\title{
Thrifty energy metabolism in catch-up growth trajectories to insulin and leptin resistance
}

\author{
Abdul G. Dulloo* \\ Department of Medicine, Division of Physiology, University of Fribourg, \\ Rue du Musée 5, CH-I 700 Fribourg, Switzerland
}

\begin{abstract}
Catch-up growth early in life (after fetal, neonatal or infantile growth retardation) is a major risk factor for later obesity, type-2 diabetes and cardiovascular diseases. These risks are generally interpreted alongside teleological arguments that environmental exposures which hinder growth early in life lead to programming of 'thrifty mechanisms' that are adaptive during the period of limited nutrient supply (or growth constraint), but which increase risks for diseases during improved nutrition and catch-up growth later in life. This paper addresses this notion of 'thrifty mechanisms' in the light of evidence that catch-up growth is characterized by a disproportionately higher rate of fat gain relative to lean tissue gain, and that such preferential catch-up fat is in part driven by energy conservation mechanisms operating via suppressed thermogenesis. It provides a molecular-physiological framework which integrates emerging insights into mechanisms by which this thrifty 'catch-up fat' phenotype cross-links with insulin and leptin resistance.
\end{abstract}

Key words: obesity; diabetes; thermogenesis; catch-up fat; thrifty phenotype.

\section{CATCH-UP GROWTH TRAJECTORIES TO OBESITY AND METABOLIC SYNDROME}

Obesity has reached epidemic proportions worldwide and is the driving force behind an equally alarming explosion of type-2 diabetes and cardiovascular diseases. Even more alarming is the fact that this rise in the global prevalence of obesity is affecting children, and increasingly preschool-age children. According to the International Obesity Task Force, at least I55 million school-age children worldwide are currently

\footnotetext{
* Corresponding author. Tel.: +4I 26300 8624; Fax: +4I 263009734.

E-mail address: abdul.dulloo@unifr.ch
} 
overweight, with 30-45 million classified as obese. In countries of the European Union alone, the number of children affected by overweight and obesity is now rising at nearly 400,000 a year, and already one in four children is overweight. These grim figures forecast a deepening crisis in public health as infants and children are being exposed to the potentially damaging metabolic and cardiovascular insults of excess fat gain during critical periods of growth and development. It is therefore not surprising that the limelight of research into understanding the pathogenesis of obesity and in developing effective strategies for its prevention has now shifted towards the identification of environmental factors, events and growth patterns that very early in life predispose to later obesity and risks for the insulin resistance (metabolic) syndrome.' In this context, catch-up growth - which has long been viewed as a physiological adaptation that re-establishes the genetically programmed growth trajectory - has emerged as a major risk factor for obesity and chronic metabolic diseases.

Indeed, the analyses of several large epidemiological databases from several countries have indicated that people who had low birth weight (a marker of fetal growth constraint) or who showed faltered growth during infancy and childhood but who subsequently showed catch-up growth, have higher susceptibility for obesity, type-2 diabetes and/or cardiovascular diseases later in life. ${ }^{2-7}$ In the most recent and most detailed retrospective longitudinal study from Finland, it was shown that men and women who were small at birth and thin during infancy and who subsequently showed catch-up growth had higher risks of coronary events associated with insulin resistance. ${ }^{7}$ These higher risks were also found to be more strongly related to the tempo of childhood gain in body mass index (BMI) than to the BMI attained at any particular age, thereby underscoring the fundamental importance of the dynamic aspects of catch-up growth per se on later insulin resistance and cardiovascular risks. There is also evidence that early postnatal catch-up growth in those born preterm also increases the risk for insulin resistance later in life. In particular, children and adolescents who showed neonatal catch-up growth after being born prematurely, irrespective of whether they were appropriate for gestational age (AGA) or small for gestational age (SGA), have reduced insulin sensitivity and increased circulating insulin levels. ${ }^{8,9}$ Furthermore, in a prospective long-term follow-up into young adulthood of men and women born very preterm, catch-up growth during early infancy was a predictor of adult fat accumulation ${ }^{10}$ as well as central fat distribution and higher insulin levels at 19 years of age." "Finally, it should be emphasized that these risks for later obesity and insulin-related complications associated with earlier catch-up growth are not confined to children born preterm or to the SGA population, but have also been reported in children born AGA but who were nonetheless relatively light and short at birth, possibly because of more subtle intrauterine growth constraint. ${ }^{5}$ The fundamental question that arises therefore is how (and why) does catch-up growth - which has long been viewed an essential feature of recovery from the deleterious effects of poor growth on development and health - confer such predisposition to obesity and insulin-related diseases?

\section{DEVELOPMENTAL PROGRAMMING AND THRIFTY PHENOTYPES}

The most common explanations are embodied in theories of metabolic 'programming' or 'imprinting', which postulate that food deprivation, malnutrition or other insults during critical periods of growth and development can lead to lasting alterations in structures and functions of tissues, and in the resetting of neuroendocrine systems 
involved in energy metabolism and weight regulation. ${ }^{12-15}$ For example, the hypothalamic-pituitary-adrenal activity, as well as the sympathoadrenal system, can be altered permanently by variations in glucocorticoid exposure due e.g. to maternal stress and maternal protein deficiency during pregnancy and lactation, all of which have been shown to lead to obesity and metabolic syndrome in animal models. ${ }^{12-15}$

A critical role for perturbations in leptin production on brain development has also been advocated in fetal and neonatal programming for obesity and the metabolic syndrome. Such a neurotrophic role for leptin early in life is strongly supported by the observations that leptin-deficient mice have reduced neural projections from the arcuate nucleus to a number of other hypothalamic nuclei involved in energy homeostasis, and that these neuroanatomical defects can be reversed by exogenous leptin administration in the neonatal period but not in adult mice. ${ }^{16}$ Of particular relevance to nutritional programming of energy balance are the findings that alterations in maternal and neonatal nutrition can alter the profile of the postnatal leptin surge and hypothalamic development. ${ }^{17}$ Indeed, intrauterine under-nutrition in mice resulted in offspring which exhibited a premature leptin surge, and subsequently showed an increased susceptibility to obesity when weaned on a high-fat diet. Thus it appears that the timing of the postnatal leptin surge plays a critical role in the development of hypothalamic circuits that influence food intake and energy expenditure, and that this process can be greatly affected by early nutrition.

The importance of leptin in developmental programming has been further strengthened by studies in rat models of maternal under-nutrition or protein restriction which have indicated that leptin administration in pregnancy and lactation protect the offspring from later obesity and insulin resistance that would otherwise develop on a high-fat diet. ${ }^{18,19}$ The clinical relevance of these observations, like the ontogeny of early-life leptin levels in humans, has yet to be established, particularly in the context that leptin is present in breast milk ${ }^{20}$ but not in infant formulae, and that breast-feeding has been found to lower the risk for obesity compared with formula feeding. ${ }^{21,22}$ Nonetheless, the findings that low leptin levels in cord blood closely reflect low adipose mass at birth, and strongly predict high rates of weight gain during infancy ${ }^{23}$, are consistent with a role for major perturbations in leptin production in developmental programming. Furthermore, the observations of high serum leptin concentrations during catch-up growth in infants born SGA ${ }^{24}$ or in children recovering from mild proteinenergy malnutrition ${ }^{25}$ raise the possibility that hyperleptinaemia and leptin resistance may play a role in the pathophysiology of catch-up growth.

Hyperinsulinemia during catch-up growth early in life, by inducing permanent changes in hypothalamic morphology and in the functional state of the sympathoadrenal system, has also been implicated in the programming of obesity later in life. ${ }^{13} \mathrm{~A}$ higher plasma insulin response to a glucose load has long been demonstrated during catch-up growth in infants born SGA ${ }^{26}$, and more recently strong associations have been described in many countries between thinness during early infancy and elevated plasma insulin during catch-up growth later in childhood. ${ }^{27,28}$ These findings, together with prospective studies from Chile ${ }^{29}$ indicating that reduced insulin sensitivity could be related to catch-up growth in SGA infants as early as at I year of age, underscore the development of insulin resistance and hyperinsulinaemia as early features of the mechanisms by which catch-up growth might confer increased risk for later diseases.

Whatever the neurohormonal mechanisms (or indeed the mechanisms for genetic imprinting) that have been proposed to explain the roles of early nutrition and growth pattern in susceptibility to later obesity and diseases, they are generally interpreted alongside the teleological arguments of an early adaptive response for a 'thrifty 
phenotype' turned maladaptive later in life. Thus, according to the thrifty phenotype hypothesis, originally put forward by Hales and Barker ${ }^{12}$, early pre- and postnatal life is a critical period during which environmental exposures that hinder growth will lead to the programming of thrifty mechanisms that is adaptive during the period of limited supply of nutrients (or growth restraint), but which contribute to increased risks for diseases during improved nutrition and catch-up growth later in life. But which components of catch-up 'growth' serve as vehicle for driving the expression of these imprinted thrifty mechanisms: catch-up in linear growth and height, catchup in lean tissue, or catch-up in body fat? In this context, there is now compelling evidence that the dynamic process of catch-up growth per se is characterized by a disproportionately higher rate of fat gain than lean tissue gain, i.e. by preferential 'catch-up fat'. ${ }^{30}$

\section{THE INSULINO-RESISTANT ‘CATCH-UP FAT’ PHENOTYPE}

This preferential catch-up fat phenotype, and its association with hyperinsulinaemia and impaired glucose homeostasis, has been documented in infants and children born SGA. In a study conducted in Switzerland ${ }^{31}$, reduced glucose oxidation and increased lipid oxidation could indeed be observed in prepubertal non-diabetic children born SGA. These impairments in substrate metabolism and in insulin sensitivity could be attributed not only to their lower lean body mass but also to their higher body fat content. More direct evidence for the occurrence of preferential catch-up fat early in life can be derived from a recent longitudinal study of body composition - assessed by dual energy $x$-ray absorptiometry (DEXA) scan - in infants between the ages of 2 and 5 years born SGA in Spain. ${ }^{32} \mathrm{~A}$ re-analysis of these data ${ }^{30}$ reveals that already between ages of 2 and 4.5 years, children born SGA gained more total body fat and less lean tissue, and hence a markedly higher ratio of fat mass to lean tissue mass, than children born AGA, despite similar gains in weight and BMI (Figure I). By about 4 years of age, those children born SGA had greater total adiposity and abdominal fat, and showed lower insulin sensitivity, than children born AGA. ${ }^{32}$ Another recent longitudinal study $^{33}$, using magnetic resonance imaging (MRI) to assess adipose tissue volume, indicated that infants born SGA and showing complete catch-up in relation to head growth and adiposity by 6 weeks after birth, still showed incomplete catch-up in length and weight. There is also accumulating evidence that the phenomenon of preferential catch-up fat also occurs during catch-up growth in infants born preterm: a population group that has been reported to show reduced insulin sensitivity and compensatory hyperinsulinaemia in childhood, adolescence and early adulthood. ${ }^{8-11}$ Indeed, the application of whole-body magnetic resonance - which allows adipose tissue imaging and its quantification - has indicated that in infants born extremely preterm $(<32$ weeks gestational age) and profoundly deficient in adipose tissue, the subsequent accelerated postnatal growth was accompanied by accelerated fat gain such that, at their expected time of delivery several weeks later, these preterm infants reached the same total adiposity as term-born infants despite the fact that they were lighter and shorter. $^{34}$ Taken together, these studies further underscore the occurrence of preferential catch-up fat, with lean tissue lagging behind in babies and infants showing catch-up growth, and their predisposition to insulin resistance later in life.

These differences in body composition, associated with impaired glucose metabolism, may persist into adulthood, as suggested by studies from Finland ${ }^{35}$ and from England using DEXA $\operatorname{scan}^{36}$, indicating that for the same BMI as age-matched controls, 

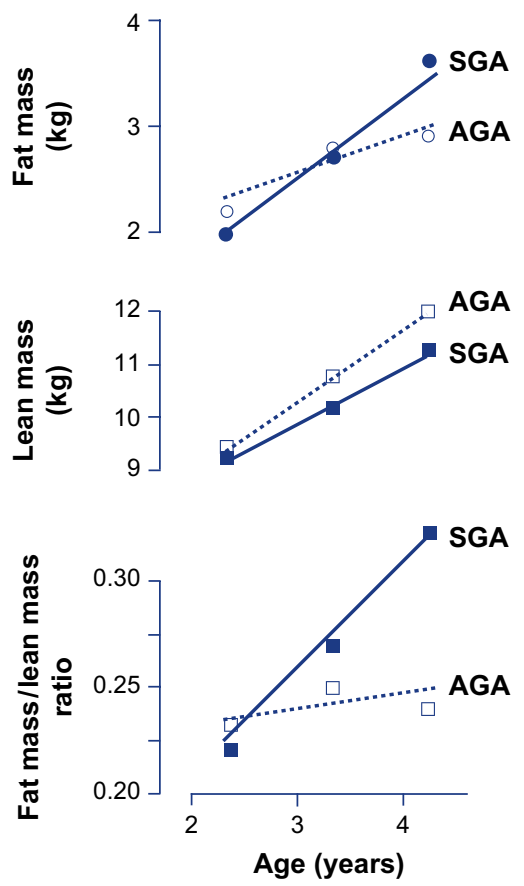

Figure I. Longitudinal studies of human body composition demonstrating preferential catch-up fat in infants born small for gestational age (SGA; $n=29)$ compared to controls born appropriate for gestational age $(A G A ; n=22)$. This figure is derived from a re-analysis of data on body weight and body composition (lean body mass and \%fat) assessed by dual energy $x$-ray absorptiometry (DEXA) scan in the study of lbanez et al ${ }^{32}$ Note that (I) the velocity of fat gain (slope of fat mass versus age) is about twofold greater in the infants born SGA than in controls born AGA, and that (2) the relatively constant ratio of fat mass to lean tissue mass in infants born AGA contrasts sharply with the marked quasi-linear increase in this ratio in infants born SGA over the 2-year period of body composition study.

healthy elderly individuals born SGA have 3-5 kg less lean tissue mass, more fat mass, and a more central fat distribution. Similarly, studies from Denmark in cohorts of healthy young men born SGA indicate that they have slightly less lean tissue mass and slightly more body fat, but clearly higher abdominal fat mass ${ }^{37}$ than age-matched controls of similar BMI, or that they showed reduced forearm glucose uptake ${ }^{38}$ and reduced muscle expression of key proteins involved in insulin signalling and glucose transport. ${ }^{39}$ Among explanations of how such non-optimal early growth followed by catch-up growth could lead to these metabolic disorders, Eriksson ${ }^{40}$ recently underscored the potential importance of impaired growth of organs and tissues that tracks into adulthood, with poor liver growth predisposing them to hepatic insulin resistance and dyslipidaemia, and poor muscle growth resulting in reduced buffering capacity for glucose disposal, and hence predisposing them towards insulin resistance. Tappy ${ }^{41}$ has proposed a model in which the metabolic disorders and impaired growth in these individuals born SGA are secondary to growth hormone resistance. According to this model, the consequential decrease in IGF-I secretion would lead to low growth rates and impaired glucose metabolism, while the increase in body fat would lead to insulin resistance through increased plasma FFAs. 
It is currently unknown whether these insulin-related impairments in people born SGA precede or are preceded by preferential abdominal fat deposition, excess circulating lipids or ectopic fat storage, all of which have been implicated in the development of insulin resistance. Nonetheless, these studies in infants, children and adults born SGA point to an early but long-lasting impairment in glucose homeostasis and body composition that can be related to preferential catch-up fat during catch-up growth. Of central importance to our understanding of the pathophysiology of catch-up growth, therefore, is the question of whether (and how) processes that regulate fat storage specifically during catch-up fat may lead to a state of insulin resistance.

\section{AUTOREGULATION OF FAT STORAGE DURING CATCH-UP FAT}

In addressing this issue, it is important to emphasize that the phenomenon of preferential catch-up fat is not limited to individuals born SGA or to the growth phase. Such increases in the ratio of fat mass to lean mass are also well documented in adults recovering body weight after weight loss due to a variety of conditions, including war-related famine, poverty-related under-nutrition, experimental starvation, anorexia nervosa and other pathophysiological 'hypermetabolic' conditions such as cancer, septic shock and AIDS. ${ }^{42}$ Thus, a common denominator in many situations where there are large decreases in body weight followed by weight recovery - whether during growth or in adulthood - is that body fat is recovered at a disproportionately faster rate than lean tissue. Since this phenomenon of preferential catch-up fat still occurs in the absence of hyperphagia and on well-balanced diets, it underscores an elevated metabolic efficiency for catch-up fat as a fundamental physiological reaction to growth retardation.

\section{Role of 'adipose-specific' suppression of thermogenesis}

There is indeed converging evidence from experimental studies of prolonged starvation and re-feeding in adult humans ${ }^{43-45}$ and in actively growing animals ${ }^{46-50}$ that an elevated efficiency for catch-up fat is a phenomenon that occurs at all ages, and that it is a carry-over effect of the suppression of thermogenesis (i.e. energy conservation mechanisms) that occurred in the preceding period of food deprivation. This has been referred to as adipose-specific control of thermogenesis ${ }^{51}$, that is, a control system that has a slow time-constant by virtue of its response to signals arising only from the state of depletion (or delayed expansion) of the body's fat stores. In this autoregulatory feedback system, signals from the depleted adipose fat stores exert a suppressive effect on thermogenesis. Support for the existence of a control system linking depletion of fat stores and suppressed thermogenesis in humans comes from the re-analysis 45,52 of longitudinal data on changes in basal metabolic rate (BMR) and in body composition from the classic 'Minnesota Experiment' of semi-starvation and re-feeding. ${ }^{43}$ In this study, 32 healthy men of normal body weight were subjected to 24 weeks of semistarvation (during which they lost $\sim 25 \%$ of initial body weight), followed by 12 weeks of restricted re-feeding on diets relatively low in fat $(\sim 20 \%$ fat by energy). As shown in Figure 2, there is a positive relationship between the deviation in body fat and the change in adjusted $B M R$, an index of altered thermogenesis calculated from the change in BMR after adjusting for losses of fat-free mass and fat mass. In other words, the greater the degree of fat depletion during starvation, the greater the reduction in adjusted BMR, and hence the greater the degree of suppressed thermogenesis. A 


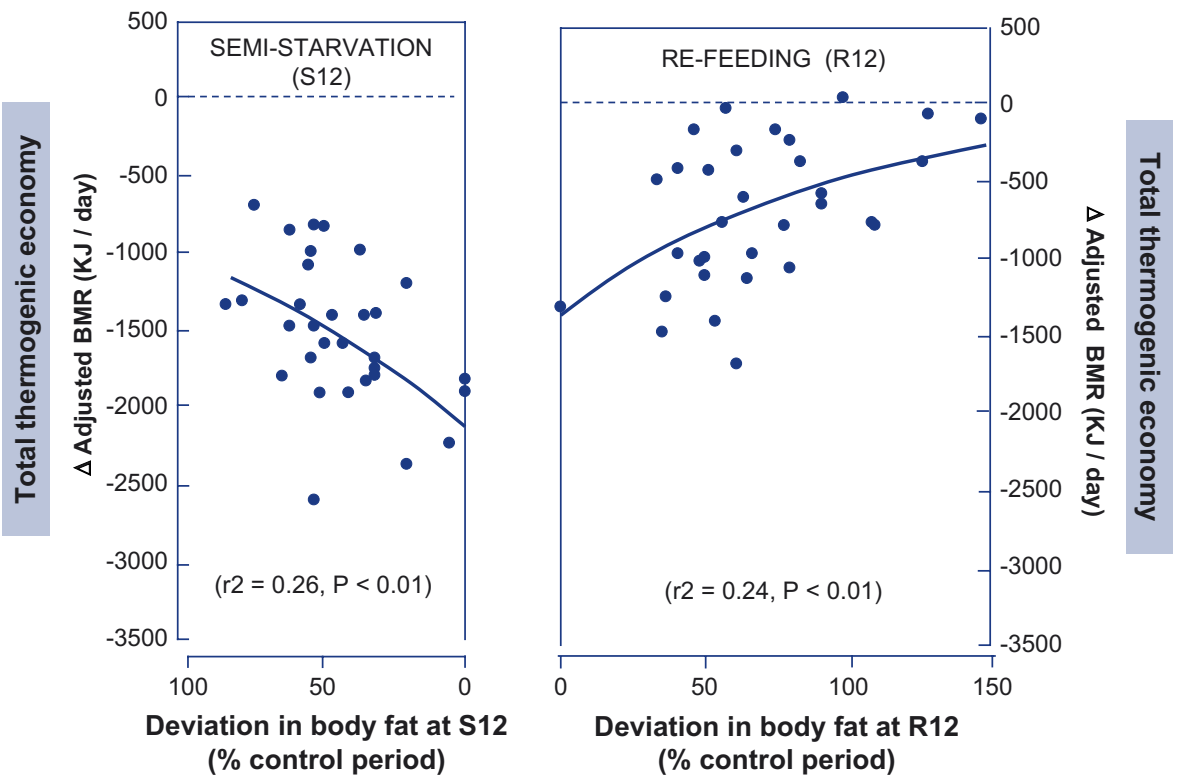

Figure 2. Minnesota experiment of human starvation and re-feeding revisited: the relationship between suppressed thermogenesis, assessed as change in basal metabolic rate (BMR) adjusted for changes in fatfree mass and fat mass, and the state of depletion of body fat stores during weight loss (SI2, week 12 of semi-starvation) and weight recovery (RI2, week 12 of restricted re-feeding). The relationship between suppressed thermogenesis and fat depletion during phases of both weight loss and weight recovery suggests the operation of a control system with a negative feedback loop between thermogenesis and the state of depletion of fat stores. Adapted from Dulloo and Jacquet (1998, American Journal of Clinical Nutrition 68: 599-606) with permission.

similar relationship was also found after the 12 -week period of restricted re-feeding, i.e., the lower the degree of fat repletion, the greater the extent of reduction in residual BMR and hence the greater the degree of reduction in thermogenesis. ${ }^{52}$ Taken together, the relationship between suppressed thermogenesis and fat depletion during phases of both weight loss and weight recovery indicates the operation of a control system with a negative feedback loop between thermogenesis and the state of depletion of fat stores.

Although a similar type of evidence linking fat depletion (or delayed expansion of the fat stores) and suppressed thermogenesis during catch-up growth in humans is not available, direct evidence for an adipose-specific suppression of thermogenesis, the role of which is to specifically accelerate body fat recovery, can be derived from studies of complete energy balance in growing rats regaining weight after semi-starvation (Figure 3). Under conditions whereby the rehabilitated animals were pair-fed to weight-matched controls, the rate of protein deposition was found to be the same as in controls, but that of fat deposition was increased by more than twofold. This was shown to be the result of 10-15\% lower energy expenditure during the first 2-3 weeks of isocaloric refeeding. ${ }^{47,48,51}$ A number of factors that could theoretically contribute to this difference in energy expenditure between re-fed and control animals (age difference, physical activity, feeding pattern) have been evaluated and shown to have a minimal impact on the difference in energy expenditure between the two 

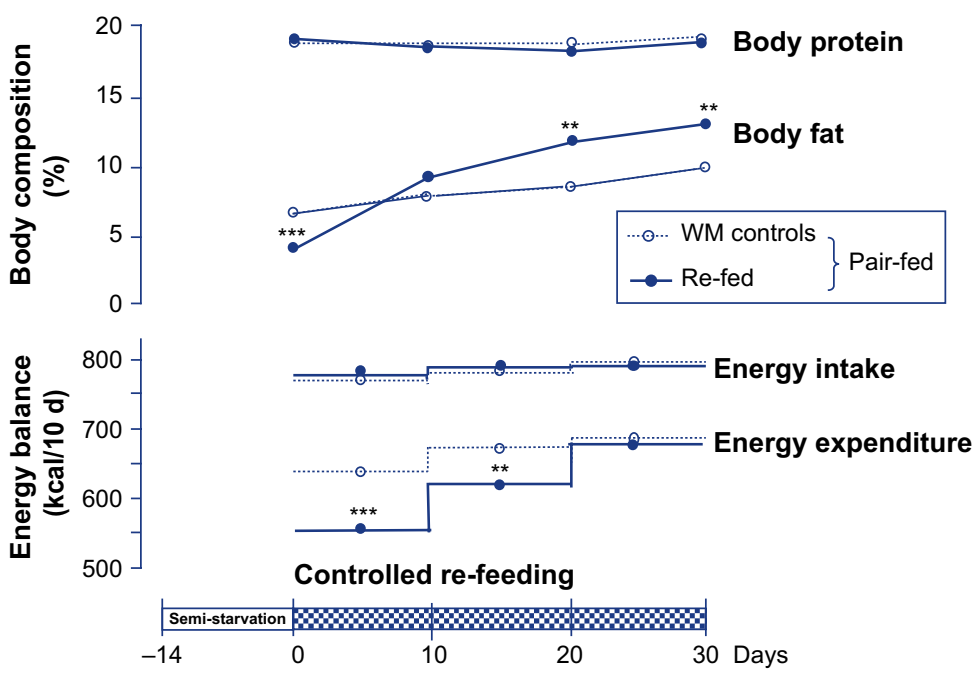

Figure 3. Sustained reduction in energy expenditure and accelerated fat deposition (catch-up fat) in a rat model of semi-starvation/re-feeding, showing preferential catch-up fat driven by suppressed thermogenesis. Changes in body composition and in energy balance were assessed over three successive periods of 10 days in re-fed animals pair-fed to weight-matched (WM) controls. The lower energy expenditure during refeeding persists for 2-3 weeks, and is due almost entirely to a sustained suppression of thermogenesis that drives catch-up fat during weight recovery. ** $P<0.01$; *** $P<0.001$ compared with WM controls. Adapted from Dulloo and Jacquet (200I, International Journal of Obesity 25 (Suppl. 5): S22-S29) with permission.

groups. Consequently, under these conditions of re-feeding, the lower energy expenditure in the re-fed animals is essentially a result of energy being spared due to sustained suppression of thermogenesis for the purpose of catch-up fat. Taken together, these findings in humans and in animals support the existence of an autoregulatory control system that participates in the regulation of catch-up growth by sustained suppression of thermogenesis, and suggest that the energy thus conserved is directed specifically for the recovery of fat mass rather than that of lean mass, i.e. a thrifty metabolism specific for preferential catch-up fat.

\section{THE THRIFTY METABOLISM THAT DRIVES CATCH-UP FAT: IMPACT ON INSULIN AND LEPTIN SENSITIVITY}

As skeletal muscle is an important site for energy conservation during starvation ${ }^{53-56}$, the control system underlying this adipose-specific control of thermogenesis could operate as a feedback loop between adipose tissue triglyceride stores and skeletal muscle metabolism. As depicted in Figure 4, it could comprise a sensor(s) of the state of depletion (or delayed expansion) of the fat stores, signal(s) dictating the suppression of thermogenesis as a function of the state of depletion of the fat stores, and an effector system mediating thermogenesis in skeletal muscle. ${ }^{57}$ At present, our understanding of the components of this system is fragmentary. However, as skeletal muscle is the major site for insulin-mediated glucose disposal, a reduction in the metabolic rate of muscle would therefore result in a reduction in glucose utilization, leading to 


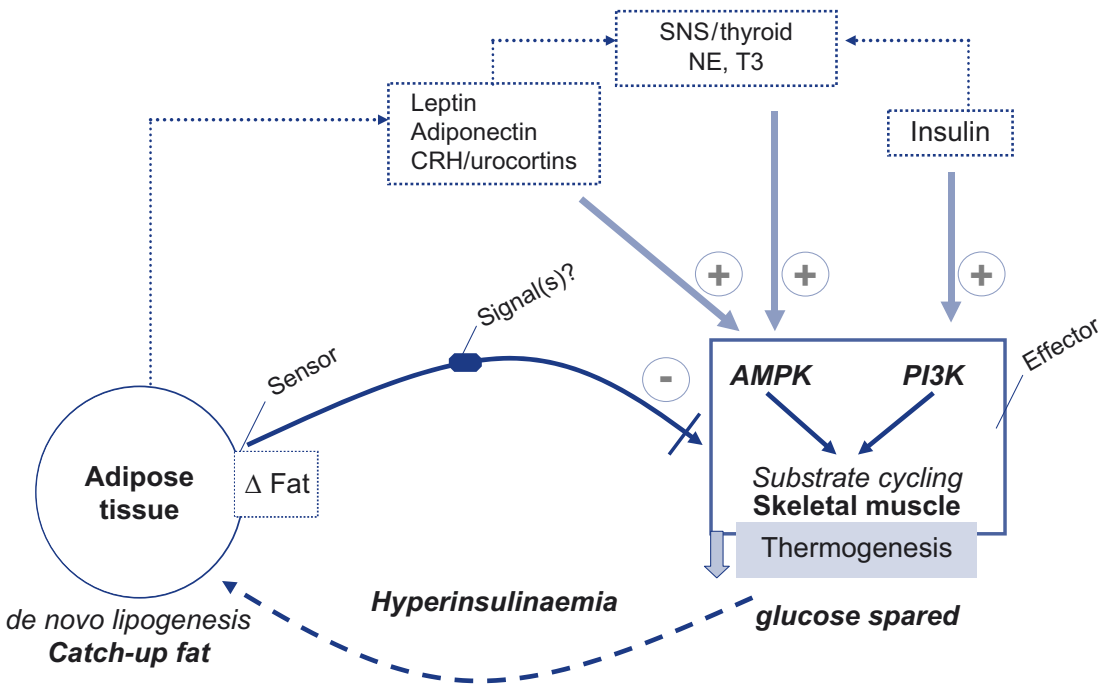

Figure 4. Schematic diagram depicting mechanisms by which the thrifty catch-up fat phenotype, driven by the adipose-specific suppression of thermogenesis, may cross-link with early development of insulin and leptin resistance. The model is built upon emerging evidence that an important thermogenic effector system in skeletal muscle is mediated by substrate cycling between de novo lipogenesis and lipid oxidation ${ }^{61,62}$, and it is orchestrated by phosphatidyloinositol 3-kinase (PI3K) and AMP-activated protein kinase (AMPK) under the influence of insulin, leptin, adiponectin and perhaps other hormones (e.g. urocortins). ${ }^{61,62,66}$ These hormones can interact to stimulate thermogenesis via the enhancement of AMPK and PI3K signalling either by acting directly on skeletal muscle or centrally through the sympathetic (SNS)/thyroid axis via norepinephrine (NE) and tri-iodothyronine (T3). By interfering with PI3K and/or AMPK signalling in skeletal muscle, the actions of the adipose-specific signal(s) that sense the state of depletion (or delayed expansion) of the adipose tissue fat stores will suppress skeletal muscle thermogenesis, which during re-feeding will lead to concomitant insulin and leptin resistance. The resulting hyperinsulinaemia serves to redirect the glucose spared from oxidation in skeletal muscle towards de novo lipogenesis and fat storage in white adipose tissue. $\mathrm{CRH}$, corticotropin releasing hormone.

hyperinsulinaemia. This in turn would serve to redirect the spared glucose towards denovo lipogenesis and fat storage in adipose tissue.

\section{Muscle-adipose glucose redistribution}

Support for this 'glucose redistribution hypothesis' can be derived from studies in our animal model of catch-up fat driven solely by suppressed thermogenesis. These have indicated that re-feeding on a low-fat diet resulted in a more pronounced elevation in plasma insulin after a glucose $\operatorname{load}^{58}$, and that under conditions of hyperinsulinaemic-euglycaemic clamps in vivo, insulin-stimulated glucose utilization in refed animals is lower in skeletal muscle but higher in white adipose tissue ${ }^{59}$, thereby suggesting a state of insulin resistance in skeletal muscle and insulin hyper-responsiveness in white adipose tissue. Furthermore, the fact that fatty acid synthase activity is higher in white adipose tissue from re-fed animals than from controls suggests enhanced conversion of glucose to lipids in the adipose tissue fat stores. ${ }^{59}$ Of particular importance in these studies comparing re-fed and control animals is that this 
redistribution of glucose utilization away from skeletal muscle towards de-novo lipogenesis and fat storage in adipose tissue can be demonstrated in the absence of between-group differences in energy intake, lean tissue mass, total body fat mass, regional fat distribution or in circulating free fatty acids. Similarly, the state of insulin resistance in skeletal muscle of the re-fed animals cannot be attributed to excess lipid storage in muscle cells, as histological staining of muscles revealed that intramyocellular lipid content in muscles from re-fed animals was not higher than in controls. ${ }^{59}$ Taken together, these data suggest that the muscle insulin resistance and adipose tissue insulin hyper-responsiveness in the re-fed animals are not related to overt lipotoxic stresses, but can be linked to the state of suppressed thermogenesis per se. In other words, the mechanisms that lead to suppressed skeletal muscle thermogenesis (in favour of catch-up fat) are interlinked with those that lead to insulin resistance during catch-up growth. By what molecular mechanisms this suppression of thermogenesis is brought about, and how these mechanisms of suppressed thermogenesis might cross-link with early development of skeletal muscle insulin resistance, are therefore fundamental issues that need to be addressed to contribute to our understanding of how catch-up growth confers increased susceptibility to insulin resistance and hyperinsulinaemia.

\section{Molecular cross-links between suppressed thermogenesis and insulin resistance}

As the concentrations of key 'adiposity' hormones that might be implicated in the link between glucose metabolism and thermogenesis in skeletal muscle (namely insulin and leptin) are rapidly restored to control levels upon transition from starvation to refeeding ${ }^{58}$, our current working hypothesis is that the suppression of thermogenesis and concomitant insulin resistance in skeletal muscle are brought about through the inhibition of mechanisms by which these (and other) hormones interact to activate thermogenesis in skeletal muscle. ${ }^{57}$ The nature of these postulated adipose-specific signal(s) that inhibit thermogenesis in skeletal muscle are unknown, but there is emerging evidence that their inhibitory actions on muscle thermogenesis could occur through interference with the activation of two major signalling pathways in substrate metabolism: phosphatidylinositol 3-kinase (PI3K) or AMP-activated protein kinase (AMPK). Indeed, ex-vivo studies of microcalorimetry in intact skeletal muscle have shown that PI3K and AMPK signalling - which are well known to influence insulin sensitivity in this tissue - are both required for hormonal stimulation of muscle thermogenesis, since the direct thermogenic effects of leptin or corticotropin-releasing hormone in skeletal muscle can be inhibited by selective inhibitors of either AMPK or PI3K. ${ }^{60-62}$ On the basis of these findings, it follows that either diminished PI3K and/or AMPK signalling in skeletal muscle could constitute impairments that are common to pathways that lead to both suppressed thermogenesis and to resistance to the actions of insulin and leptin during catch-up growth (Figure 4). This notion is indeed supported by recent data from our laboratory ${ }^{63}$ indicating diminished basal and insulin-stimulated PI3K activity in skeletal muscle of rats showing catch-up fat driven by suppressed thermogenesis during re-feeding on a low-fat diet, with additional impairments in leptin-induced activation of AMPK during isocaloric re-feeding on diets high in saturated fats.

Which molecular effectors of skeletal muscle thermogenesis might be under the control of PI3K and AMPK signalling, and what factors contribute to such early 
impairments in these signalling pathways (i.e. impairments that precede excess adiposity, circulating free fatty acids or intramyocellular lipids) are questions that have only recently been the subject of investigation. Of particular interest is the demonstration that rats showing catch-up fat due to suppressed thermogenesis also show diminished mitochondrial mass and oxidative capacity specifically in the subsarcolemmal compartment of skeletal muscle. ${ }^{64}$ Given the important role that subsarcolemmal mitochondria have for bioenergetic support of signal transduction and substrate transport ${ }^{65}$, the possibility arises that deficiency in this subpopulation of mitochondria could contribute to impaired PI3K and AMPK signalling. While a reduction in the number of subsarcolemmal mitochondria per se could contribute to the suppression of thermogenesis that favours catch-up fat, impairments in PI3K or in AMPK could also lead to a slowdown of a 'futile' substrate cycling between de-novo lipogenesis and lipid oxidation. This thermogenic effector has recently been implicated in hormonal control of skeletal muscle thermogenesis, with $\mathrm{PI} 3 \mathrm{~K}$ being required in controlling the flux of substrates through de-novo lipogenesis, and AMPK being required in controlling the flux of de-novo-synthesized fatty acids through mitochondrial $\beta$-oxidation. ${ }^{61,62,66}$

Overall, therefore, there is emerging evidence for insulin and leptin resistance in the early phase of catch-up fat, and for impairments in PI3K and AMPK signalling in skeletal muscle in the mechanisms by which suppressed thermogenesis might crosslink with insulin and leptin resistance during catch-up growth. Consequently, impairments in these signalling pathways in skeletal muscle may constitute the molecular basis by which the thrifty metabolism (suppressed thermogenesis) that accelerates fat recovery confers enhanced susceptibility to the development of insulin resistance and hyperinsulinaemia during catch-up growth.

\section{THRIFTY METABOLISM DRIVING CATCH-UP FAT: ADAPTIVE TURNED MALADAPTIVE}

The biological significance of this thrifty metabolism underlying catch-up fat - characterized by concurrent hyperinsulinaemia, skeletal muscle insulin and leptin resistance, and adipose tissue insulin hyper-responsiveness - would be to achieve both blood glucose homeostasis and the rapid replenishment of the fat stores by diverting glucose spared from oxidation in skeletal muscle to lipogenesis and fat storage in adipose tissue. Under conditions of intermittent periods of food availability that prevailed during much of mammalian evolution, this coordinated redistribution of glucose from muscle to adipose tissue probably had survival value since it enables the rapid replenishment of fat stores, and hence rapid restoration of survival capacity, without compromising blood glucose homeostasis. Despite its 'adaptive' nature within the context of a lifestyle of famine-and-feast, this state may have deleterious consequences in the context of the modern lifestyle, characterized by low physical activity and energy-dense diets rich in fat and refined carbohydrates. In fact, a shift in diet from complex carbohydrates to animal fat and refined carbohydrates leads to an exacerbated suppression of thermogenesis, a more pronounced state of hyperinsulinaemia, hyperglycaemia and excess adiposity in animals recovering weight, compared with fed controls growing spontaneously on isocaloric amounts of the same high-fat diet. $^{58}$ As depicted in Table I, high levels of (saturated) fat in the diet, even in the absence of hyperphagia, has compromised a complex homeostatic system. 


\begin{tabular}{|c|c|c|}
\hline & \multicolumn{2}{|c|}{ Isocaloric re-feeding after semi-starvation } \\
\hline & $\begin{array}{l}\text { Low-fat diet } \\
\text { (RF-LF versus C-LF) }\end{array}$ & $\begin{array}{c}\text { High-fat diet } \\
\text { (RF-HF versus C-HF) }\end{array}$ \\
\hline Thermogenesis & $\downarrow$ & $\downarrow \downarrow$ \\
\hline Rate of body fat gain & $\uparrow$ & $\uparrow \uparrow$ \\
\hline Plasma leptin & $\uparrow$ & $\uparrow \uparrow$ \\
\hline Plasma insulin & $\uparrow$ & $\uparrow \uparrow$ \\
\hline Glucose tolerance & Normal (euglycaemic) & Impaired (hyperglycaemic) \\
\hline
\end{tabular}

To what extent this disruption of glycaemia resides in an exacerbated state of skeletal-muscle insulin resistance and/or in the inability of adipose tissue to dispose of excess glucose spared as a result of an exacerbated suppression of thermogenesis during high-fat re-feeding is currently under investigation. Whatever the answers to these questions, however, it is clear that as the phase of catch-up growth may last for several months to several years in humans ${ }^{7}$, the drive to suppressed thermogenesis for the purpose of sparing glucose for catch-up fat, via its orchestration through insulin and leptin resistance in skeletal muscle together with altered insulin responsiveness in adipose tissue, constitute a thrifty phenotype that predisposes individuals with catch-up growth to insulin and leptin resistance and risks for later metabolic syndrome.

Could such thrifty energy metabolism in humans be programmed or imprinted earlier in fetal or neonatal life, and hence long-lasting so as to operate beyond the phase of catch-up growth? The answer to this question seems plausible in the light of recent evidence, depicted in Figure 5, indicating a lower resting energy expenditure (even after adjustments for weight, height, or lean tissue mass) in a group of elderly adults with low birth weight and showing a twofold greater prevalence of metabolic syndrome than in those with a high birth weight. ${ }^{67}$ In the complex interactions between the genetic constitution of the individual, programming or imprinting early in life, and a subsequent lifestyle of energy-dense foods and low physical activity, the thrifty 'catch-up fat' phenotype - which evolved to increase survival capacity in a hunter-gatherer lifestyle of periodic food shortages - could thus be a central event in growth trajectories to obesity and to diseases that cluster into the insulin resistance (metabolic) syndrome. Thus, the mechanisms by which body fat is acquired during early growth would seem to be at least as important as the consequences of excess fat per se in the pathogenesis of type-2 diabetes and cardiovascular diseases.

\section{SUMMARY}

Epidemiological and clinical studies have suggested that neonates, infants and children who show catch-up growth are predisposed to obesity, type-2 diabetes and cardiovascular diseases later in life. The pathophysiological mechanisms by which catch-up growth confer increased risks for these diseases are obscure, but there is compelling 


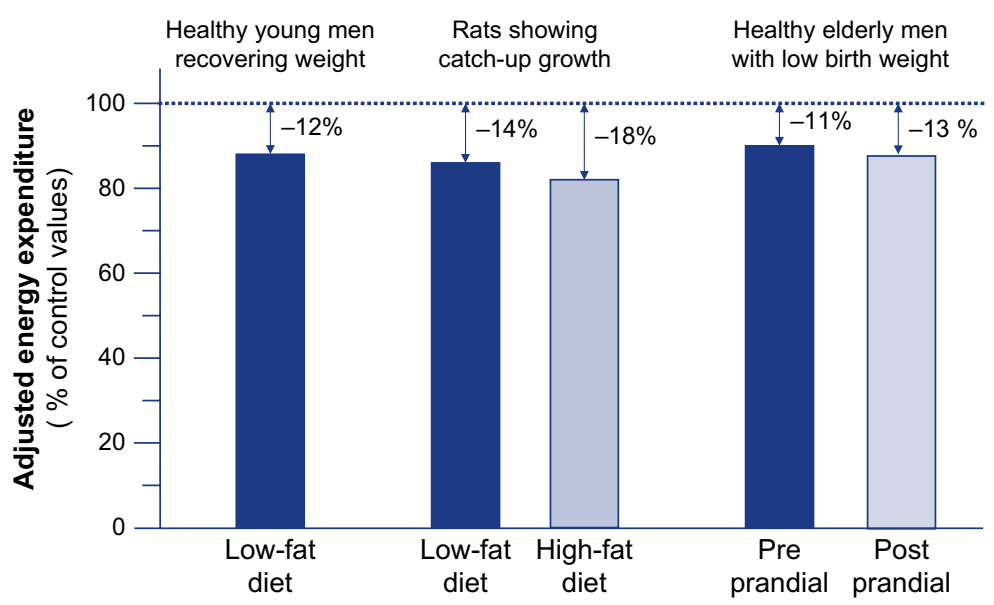

Figure 5. Extent of suppressed thermogenesis (thrifty energy metabolism) in the compartments of resting energy expenditure (REE) in young men or rats showing preferential catch-up fat after semi-starvation, as well as in weight-stable healthy elderly men with low birth weight. The reduction in adjusted REE is relative to control values set at $100 \%$ (dotted line). The following should be noted. (I) The value for extent of suppressed thermogenesis (as \% of baseline basal metabolic rate) in healthy young men recovering body weight after semi-starvation is derived from the Minnesota Experiment of Keys et al ${ }^{43}$, and was determined in a reanalysis of the data on their basal metabolic rate after adjustments for changes in fat-free mass and in fat mass relative to pre-starvation values. ${ }^{52}$ (2) In rats showing catch-up growth after post-weaning semi-starvation, REE is taken as their total energy expenditure (essentially sedentary) relative to that of weight-matched controls consuming the same amount of energy and gaining protein mass at the same rate, whether during re-feeding on a low-fat diet or on an isocaloric diet rich in animal saturated fat. ${ }^{47,48,58}$ (3) In the group of healthy elderly men with low birth weight relative to another group with high birth weight, REE was determined from measurements of both preprandial and postprandial REE adjusted for body weight and height; in this study by Kensara et al, the adjustments of REE for fat-free mass, muscle mass, non-muscular (fat-free soft tissue) mass, or for the ratio of muscle to non-muscle (fat-free soft tissue) mass also yielded lower REE values (by $8-12 \%$ ) in the low-birth-weight group than in the high-birth-weight group.

evidence that the dynamic process of catch-up growth per se is characterized by insulin resistance and by a disproportionately higher rate in recovering body fat than lean tissue (i.e. preferential 'catch-up fat'). This chapter first focuses upon this preferential 'catch-up fat' phenotype as a risk factor for obesity and insulin-related complications in infants and children who experienced catch-up growth after earlier fetal or neonatal growth retardation, or after preterm birth. It subsequently reviews the evidence indicating that such preferential catch-up fat is primarily driven by energy conservation (thrifty) mechanisms operating via suppressed thermogenesis, with glucose thus spared from oxidation in skeletal muscle being directed towards de novo lipogenesis and storage in adipose tissue. A molecular-physiological framework is presented which integrates emerging insights into the mechanisms by which this thrifty 'catch-up fat' phenotype cross-links with early development of insulin and leptin resistance. In the complex interactions between the genetic constitution of the individual, programming earlier in life, and a subsequent lifestyle of energy-dense foods and low physical activity, this thrifty 'catch-up fat' phenotype - which probably evolved to increase survival capacity in a hunter-gatherer lifestyle of periodic food shortages - is a central event in growth trajectories to obesity and the insulin resistance (metabolic) syndrome. 


\section{Practice points}

- a pattern of growth characterized by postnatal catch-up growth has emerged as a major factor that predisposes to later obesity, type-2 diabetes, and cardiovascular diseases

- the development of insulin resistance, associated with a disproportionate rate in recovering adipose tissue relative to lean tissue (i.e. preferential catch-up fat), are early features of the mechanisms by which catch-up growth confers increased risk for these chronic diseases

- the regulation of fat storage through suppressed thermogenesis seems to be intimately linked to insulin resistance during catch-up growth

- how body fat is acquired during early growth would seem to be at least as important as the consequences of excess fat per se in the pathogenesis of type-2 diabetes and cardiovascular disease

\section{Research agenda}

- the ontogeny of early-life leptin in humans, and the clinical relevance of breastmilk leptin in growth and development, need to be thoroughly investigated

- how suppressed thermogenesis might cross-link with insulin resistance in skeletal muscle are fundamental issues that need to be addressed in order to understand how catch-up growth confers increased susceptibility to glucose intolerance

- to what extent (and by what mechanisms) the disruption of glycaemia during catch-up growth might also reside in the inability of adipose tissues to dispose of excess glucose needs to be investigated

- there is a need to develop nutritional strategies for counteracting the state of hyperinsulinaemia and excessive fat recovery (while improving lean tissue deposition) during catch-up growth through coordinated basic and clinical research

\section{ACKNOWLEDGEMENTS}

This work is funded by the Swiss National Science Research Foundation (grant \# 3200B0-I I3634).

\section{REFERENCES}

I. Dulloo AG, Antic V, Yang $Z$ et al. Propellers of growth trajectories to obesity and the metabolic syndrome. International Journal of Obesity 2006; 30: SI-S3.

2. Eriksson JG, Forsen T, Tuomilehto J et al. Catch-up growth in childhood and death from coronary heart disease: longitudinal study. BMJ 1999; 3 18: 427-43I. 
3. Cianfarani S, Germani D \& Branca F. Low birth weight and adult insulin resistance: the 'catch-up growth' hypothesis. Archives of Diseases in Childhood. Fetal Neonatal Edition 1999; 8I: F7I-F73.

4. Huxley RR, Shiell AW \& Law CM. The role of size at birth and postnatal catch-up growth in determining systolic blood pressure: a systematic review of the literature. Journal of Hypertension 2000; 18: 8I5-83I.

5. Ong KK, Ahmed ML, Emmett PM et al. Association between postnatal catch-up growth and obesity in childhood: prospective cohort study. BMJ 2000; 320: 967-97I.

6. Levy-Marchal C, Jaquet D \& Czernichow P. Long-term metabolic consequences of being born small for gestational age. Seminars in Neonatology 2000; 9: 67-74.

*7. Barker DJ, Osmond C, Forsen TJ et al. Trajectories of growth among children who have coronary events as adults. The New England Journal of Medicine 2005; 353: 1802-1809.

8. Singhal A, Fewtrell M, Cole TJ et al. Low nutrient intake and early growth for later insulin resistance in adolescents born preterm. Lancet 2003; 361(9363): 1089-1097.

*9. Hofman PL, Regan F, Jackson WE et al. Premature birth and later insulin resistance. The New England Journal of Medicine 2004; 35I: 2179-2186.

10. Euser AM, Finken MJ, Keijzer-Veen MG et al. Associations between prenatal and infancy weight gain and BMI, fat mass, and fat distribution in young adulthood: a prospective cohort study in males and females born very preterm. The American Journal of Clinical Nutrition 2005; 8I: 480-487.

II. Finken MJ, Keijzer-Veen MG, Dekker FW et al. Preterm birth and later insulin resistance: effects of birth weight and postnatal growth in a population based longitudinal study from birth into adult life: insulin resistance 19 years after preterm birth. Diabetologia 2006; 49: 478-485.

12. Hales CN \& Barker DJ. The thrifty phenotype hypothesis. British Medical Bulletin 200I; 60: 5-20.

*13. Young JB. Developmental origins of obesity: a sympathoadrenal perspective. International Journal of Obesity 2006; 30: S4I-S49.

*14. Cottrell EC \& Ozanne SE. Developmental programming of energy balance and the metabolic syndrome. The Proceedings of the Nutrition Society 2007; 66: 198-206.

*15. Stocker CJ, Arch JRS \& Cawthorne MA. Fetal origins of insulin resistance and obesity. The Proceedings of the Nutrition Society 2005; 64: |43-15I.

16. Bouret SG, Draper SJ \& Simerly RB. Trophic action of leptin on hypothalamic neurons that regulate feeding. Science 2004; 304: 108-I I0.

17. Yura $\mathrm{S}$, Itoh $\mathrm{H}$, Sagawa $\mathrm{N}$ et al. Role of premature leptin surge in obesity resulting from intrauterine under-nutrition. Cell Metabolism 2005; I: 37I-378.

18. Stocker C, O'Dowd J, Morton NM et al. Modulation of susceptibility to weight gain and insulin resistance in low birthweight rats by treatment of their mothers with leptin during pregnancy and lactation. International Journal of Obesity 2004; 28: I29-136.

19. Vickers MH, Gluckman PD, Coveny AH et al. Neonatal leptin treatment reverses developmental programming. Endocrinology 2005; 146: 42।I-42I6.

20. Houseknecht KL, McGuire MK, Portocarrero CP et al. Leptin is present in human milk and is related to maternal plasma leptin concentration and adiposity. Biochemical and Biophysical Research Communications 1997; 240: 742-747.

21. Locke R. Preventing obesity: the breast milk-leptin connection. Acta Paediatrica 2002; 91: 89I-894.

22. Dewey KG. Is breastfeeding protective against child obesity? Journal of Human Lactation 2003; 19: 9-18.

23. Ong KK, Ahmed ML, Sherriff A et al. Cord blood leptin is associated with size at birth and predicts infancy weight gain in humans. ALSPAC Study Team. Avon Longitudinal Study of Pregnancy and Childhood. The Journal of Clinical Endocrinology and Metabolism 1999; 84: II45-I I 48.

*24. Jaquet D, Leger J, Tabone MD et al. High serum leptin concentrations during catch-up growth of children born with intrauterine growth retardation. The Journal of Clinical Endocrinology and Metabolism 1999; 84: 1949-1953.

25. Buyukgebiz B, Ozturk Y, Yilmaz S et al. Serum leptin concentrations in children with mild protein-energy malnutrition and catch-up growth. Pediatrics International 2004; 46: 534-538.

26. Colle $E$, Schiff $D$, Andrew $G$ et al. Insulin responses during catch-up growth of infants who were small for gestational age. Pediatrics 1976; 57: 363-37I.

27. Crowther NJ, Cameroun N, Trusler J et al. Association between poor glucose tolerance and rapid post natal weight gain in seven-year-old children. Diabetologia 1998; 41: II63-II67.

*28. Ong KK \& Dunger DB. Birth weight, infant growth and insulin resistance. European Journal of Endocrinology 2004; I 5 I (Suppl. 3): UI3I-UI39. 
29. Soto N, Bazaes RA, Pena $V$ et al. Insulin sensitivity and secretion are related to catch-up growth in small-for-gestational-age infants at age I year: results from a prospective cohort. The Journal of Clinical Endocrinology and Metabolism 2003; 88: 3645-3650.

30. Dulloo AG, Jacquet J, Seydoux J et al. The thrifty 'catch-up fat' phenotype: its impact on insulin sensitivity during growth trajectories to obesity and metabolic syndrome. International Journal of Obesity 2006; 30(Suppl. 4): S23-S35.

3I. Jornayvaz FR, Selz R, Tappy L et al. Metabolism of oral glucose in children born small for gestational age: evidence for an impaired whole body glucose oxidation. Metabolism 2004; 53: 847-85I.

32. Ibanez L, Ong K, Dunger DB et al. Early development of adiposity and insulin resistance after catch-up weight gain in small-for-gestational-age children. The Journal of Clinical Endocrinology and Metabolism 2006; 91 : 2I53-2I58.

33. Modi N, Thomas EL, Harrington TA et al. Determinants of adiposity during preweaning postnatal growth in appropriately grown and growth-restricted term infants. Pediatric Research 2006; 60: 345348.

34. Uthaya S, Thomas EL, Hamilton G et al. Altered adiposity after extremely preterm birth. Pediatric Research 2005; 57: 211-2I5.

35. Eriksson J, Forsen T, Tuomilehto J et al. Size at birth, fat-free mass and resting metabolic rate in adult life. Hormone and Metabolic Research 2002; 34: 272-276.

36. Kensara OA, Wootton SA, Phillips DI et al. Fetal programming of body composition: relation between birth weight and body composition measured with dual-energy X-ray absorptiometry and anthropometric methods in older Englishmen. The American Journal of Clinical Nutrition 2005; 82: 980-987.

37. Rasmussen EL, Malis C, Jensen CB et al. Altered fat tissue distribution in young adult men who had low birth weight. Diabetes Care 2005; 28: I5I-I53.

38. Hermann TS, Rask-Madsen $\mathrm{C}$, Ihlemann $\mathrm{N}$ et al. Normal insulin-stimulated endothelial function and impaired insulin-stimulated muscle glucose uptake in young adults with low birth weight. The Journal of Clinical Endocrinology and Metabolism 2003; 88: I252-I 257.

39. Ozanne SE, Jensen $\mathrm{CB}$, Tingey $\mathrm{KJ}$ et al. Low birthweight is associated with specific changes in muscle insulin-signalling protein expression. Diabetologia 2005; 48: 547-552.

40. Eriksson JG. Early growth, and coronary heart disease and type 2 diabetes: experiences from the Helsinki Birth Cohort Studies. International Journal of Obesity 2006; 30: SI8-S22.

4I. Tappy L. Adiposity in children born small for gestational age. International Journal of Obesity 2006; 30: S36-S40.

42. Dulloo AG, Jacquet J \& Montani JP. Pathways from weight fluctuations to metabolic diseases: focus on maladaptive thermogenesis during catch-up fat. International Journal of Obesity 2002; 26(Suppl. 2): S46-S57.

43. Keys A, Brozek J, Henschel A et al. The Biology of Human Starvation. Minneapolis, MN: University of Minnesota Press, 1950.

44. Weyer C, Walford RL, Harper IT et al. Energy metabolism after 2 y of energy restriction: the Biosphere 2 experiment. The American Journal of Clinical Nutrition 2000; 72: 946-953.

45. Dulloo AG, Jacquet J \& Girardier L. Autoregulation of body composition during weight recovery in humans: the Minnesota Experiment revisited. International Journal of Obesity 1996; 20: 393-405.

46. Hill JO, Fried SK \& Digirolamo M. Effects of fasting and restricted refeeding on utilization of ingested energy in rats. The American Journal of Physiology 1984; 247: R318-R327.

47. Dulloo AG \& Girardier L. Adaptive changes in energy expenditure during refeeding following low calorie intake: evidence for a specific metabolic component favouring fat storage. The American Journal of Clinical Nutrition 1990; 52: 4I5-420.

48. Dulloo AG \& Girardier L. Adaptive role of energy expenditure in modulating body fat and protein deposition during catch-up growth after early under-nutrition. The American Journal of Clinical Nutrition 1993; 58: 614-621.

49. Evans SA, Messina MM, Knight WD et al. Long-Evans and Sprague-Dawley rats exhibit divergent responses to refeeding after caloric restriction. The American Journal of Physiology 2005; 288: RI468RI476.

50. MacLean PS, Higgins JA, Johnson GC et al. Enhanced metabolic efficiency contributes to weight regain after weight loss in obesity-prone rats. The American Journal of Physiology 2004; 287: RI306-RI3I5.

5I. Dulloo AG \& Jacquet J. An adipose-specific control of thermogenesis in body weight regulation. International Journal of Obesity 200I; 25(Suppl. 5): S22-S29. 
52. Dulloo AG \& Jacquet J. Adaptive reduction in basal metabolic rate in response to food deprivation in humans: a role for feedback signals from fat stores. The American Journal of Clinical Nutrition 1998; 68: 599-606.

53. Foster GD, Wadden TA, Kendrick ZV et al. The energy cost of walking before and after significant weight loss. Medicine and Science in Sports and Exercise 1995; 27: 888-894.

54. Kulkarni R \& Shetty P. Net mechanical efficiency during stepping in chronically energy deficient human subjects. Annals of Human Biology 1992; 19: $421-425$.

55. Rosenbaum M, Vandenborne K, Goldsmith R et al. Effects of experimental weight perturbation on skeletal muscle work efficiency in human subjects. The American Journal of Physiology 2003; 285: RI83-RI92.

56. Ma SW \& Foster DO. Starvation-induced changes in metabolic rate, blood flow, and regional energy expenditure in rats. Canadian Journal of Physiology and Pharmacology 1986; 64: 1252-1258.

57. Dulloo AG. A role for suppressed skeletal muscle thermogenesis in pathways from weight fluctuations to the insulin resistance syndrome. Acta Physiologica Scandinavica 2005; 184: 295-307.

58. Crescenzo R, Samec S, Antic V et al. A role for suppressed thermogenesis favouring catch-up fat in the pathophysiology of catch-up growth. Diabetes 2003; 52: 1090-1097.

*59. Cettour-Rose P, Samec S, Russell AP et al. Redistribution of glucose from skeletal muscle to adipose tissue during catch-up fat: a link between catch-up growth and later metabolic syndrome. Diabetes 2005; 54: 75I-756.

60. Dulloo AG, Stock MJ, Solinas G et al. Leptin directly stimulates thermogenesis in skeletal muscle. FEBS Letters 2002; 5 I 5: 109-113.

61. Solinas G, Summermatter S, Mainieri D et al. The direct effect of leptin on skeletal muscle thermogenesis is mediated by substrate cycling between de novo lipogenesis and lipid oxidation. FEBS Letters 2004; 577: 539-544.

62. Solinas G, Summermatter S, Mainieri D et al. Corticotropin-releasing hormone directly stimulates thermogenesis in skeletal muscle possibly through substrate cycling between de novo lipogenesis and lipid oxidation. Endocrinology 2006; I47: 31-38.

63. Summermatter S, Mainieri D, Russell AP et al. Skeletal muscle insulin and leptin resistance during early catch-up fat driven by suppressed thermogenesis: A role for impaired PI3K and AMPK signalling. International Journal of Obesity 2006; 30: S70.

64. Crescenzo R, Lionetti L, Mollica MP et al. Altered skeletal muscle subsarcolemmal mitochondrial compartment during catch-up fat after caloric restriction. Diabetes 2006; 55: 2286-2293.

65. Hood D. Plasticity in skeletal, cardiac, and smooth muscle: contractile activity-induced mitochondrial biogenesis in skeletal muscle. Journal of Applied Physiology 200 I; 90: II37-II57.

*66. Dulloo AG, Gubler M, Montani JP et al. Substrate cycling between de novo lipogenesis and lipid oxidation: a thermogenic mechanism against skeletal muscle lipotoxicity and glucolipotoxicity. International Journal of Obesity 2004; 28(Suppl. 4): S29-S37.

*67. Kensara OA, Wootton SA, Phillips DI et al. Substrate-energy metabolism and metabolic risk for cardiovascular disease in relation to fetal growth and adult body composition. The American Journal of Physiology 2006; 29 I: E365-E37I. 